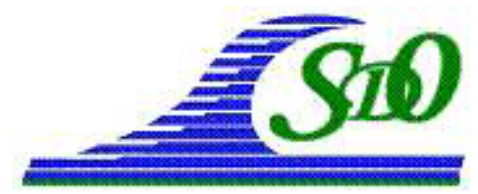

XI ìmes Journées Nationales Génie Côtier - Génie Civil

Les Sables d'Olonne, 22-25 juin 2010

DOI:10.5150/jngcgc.2010.009-C C Editions Paralia CFL

disponible en ligne - http://www.paralia.fr - available online

\title{
Simulation en méthode particulaire des sollicitations hydrodynamiques d'ouvrages marins
}

\author{
Jean-Marc CHERFILS ${ }^{1}$, Louis BLONCE ${ }^{1}$, \\ Grégory PINON ${ }^{1}$, Elie RIVOALEN ${ }^{2}$
}

\author{
1. Université du Havre, Laboratoire Ondes \& Milieux Complexes, FRE 3102 CNRS, \\ 53 rue Prony, 76058 Le Havre, France. \\ jean-marc.cherfils@univ-lehavre.fr \\ 2. Institut National des Sciences Appliquées de Rouen, \\ Laboratoire de Mécanique de Rouen, EA 3828, \\ Avenue de l'Université, BP 08, 76801 St Étienne du Rouvray, France.
}

\section{Résumé :}

Notre étude porte sur l'amortissement d'une houle régulière par une plaque horizontale immergée en canal. L'interaction non-linéaire entre l'onde de gravité, la plaque et la surface libre conduit à un transfert d'une partie de l'énergie de la houle incidente vers ses harmoniques, ainsi qu'à la création périodique de tourbillons aux extrémités. Ainsi, un code de simulation en méthode particulaire SPH (Smoothed Particle Hydrodynamics) a été développé pour l'étude des interactions fluide-structure dans le domaine du génie côtier, permettant la mesure de données physiques très ciblées et souvent inaccessibles expérimentalement.

Nativement, la méthode SPH est bien adaptée au suivi de déformations complexes d'interfaces, ce qui en fait un outil précis pour la modélisation de la houle jusqu'au déferlement. De nombreuses améliorations ont également été apportées afin d'améliorer la prise en compte d'écoulements à la dynamique plus lente (interaction houle-ouvrages marins). Une parallélisation efficace a d'ailleurs été développée, permettant la simulation d'écoulements bi- et tridimensionnels moyennant des temps de calcul raisonnables. Enfin, une nouvelle technique, inspirée de la méthode des frontières immergées, est employée pour la prise en compte des conditions aux limites de type adhérence sur les parois des ouvrages.

\section{Mots-clés :}

Méthode particulaire - SPH - Obstacle immergé - Protection côtière - Conditions aux limites

\section{Introduction}

La méthode SPH (Smoothed Particle Hydrodynamics) a initialement été développée pour la simulation de systèmes gravitaires en astrophysique (GINGOLD \& MONAGHAN, 1977 ; LUCY, 1977). Par sa nature lagrangienne et sans maillage, SPH est particulièrement adaptée aux simulations en domaine ouvert et a connu un vif succès 
dans ce type d'applications. L'état du système y est représenté par un ensemble de particules possédant leurs propres propriétés physiques et se déplaçant selon un système d'équations de conservation établi. On obtient alors une approximation numérique de la solution de ce système. Une extension de la méthode à l'hydrodynamique a ensuite été proposée (MONAGHAN, 1994). SPH a été appliquée à un grand nombre de problèmes différents, dominés par des phénomènes non-linéaires et de grandes déformations du milieu, où l'absence de maillage devient intéressante: les écoulements diphasiques, les écoulements de fluides visqueux, les écoulements en milieux poreux, les simulations d'impacts ou d'explosions.

Nous proposons ici une application de la méthode à l'étude de l'interaction fluidestructure générée par la propagation d'une onde de gravité dans un canal ouvert contenant une plaque horizontale immergée. Ce type de structure a déjà inspiré des projets réels d'amortissement de la houle, près de côtes (plan d'eau aux jeux olympiques de Barcelone, 1992 ; digue flottante à Monaco, 2002 ; tables conchylicoles). La nécessité de prendre en compte d'importantes déformations de la surface libre, des problèmes d'impacts et la possibilité de traiter simplement différentes phases fluides (air, eau, fond sédimentaire, voir figure 1) ont justifié le choix de cette méthode.

D'abord, le code de calcul et ses fonctionnalités seront présentés sur le cas test, largement étudié dans la bibliographie, de l'effondrement d'une masse d'eau et de son impact sur un mur vertical. Les résultats seront comparés aux données expérimentales et les performances seront exposées.

Nous traiterons enfin le problème de la plaque immergée en montrant la capacité du modèle à prendre en compte des écoulements complexes autour d'obstacles et à décrire l'évolution de grandeurs physiques liées à l'écoulement (vitesse, pression, surface libre) ou les effets d'interactions entre la plaque et la houle (taux de réflexion).

\section{Modèle}

Le code de calcul présenté ici permet la simulation des équations de Navier-Stokes (ou Euler si on néglige la viscosité) discrétisée selon la méthode SPH, qui s'appuie sur la notion d'opérateur-intégrale pour l'approximation des grandeurs transportées par les particules et de leurs dérivées.

\subsection{Schéma d'interpolation}

L'opérateur-intégrale permettant l'interpolation d'un champ scalaire $f$, à partir des données connues sur les particules, s'écrit comme le produit de convolution suivant :

$$
\left\langle f_{i}\right\rangle=\int_{\Omega} f(x) W\left(x_{i}-x, h\right) d x
$$

où $x_{i}$ est la position de la $i^{\text {ème }}$ particule. $W\left(x_{i}-x, h\right)$ est un noyau d'interpolation de pas $h$. Le noyau doit respecter les conditions suivantes afin d'assurer la convergence du schéma d'interpolation : 
$\int_{\Omega} W\left(x_{i}-x, h\right) d x=1$

$\lim _{h \rightarrow 0} W\left(x_{i}-x, h\right)=\delta\left(x_{i}-x\right)$

L'équation (2) représente la condition de normalisation du noyau tandis que l'équation (3) décrit le fait que le noyau doive tendre vers la mesure de Dirac quand son pas tend vers 0 . Le noyau, respectant ces conditions, employé ici est une fonction gaussienne (COLAGROSSI \& LANDRINI, 2003), à symétrie radiale (voir équation (4)), modifiée afin de permettre une coupure au-delà d'une distance de $3 h$. Cette propriété de compacité ajoutée au noyau autorise l'apport d'un certain nombre d'optimisations développées plus loin.

Le pas d'interpolation $h$ est défini en fonction de l'espacement initial entre particules $\Delta x$ par la relation suivante :

$h=\kappa \Delta x$

avec $\kappa=1,33$, ce qui donne environ 50 particules dans le voisinage de la particule d'intérêt. En dérivant l'équation (1), puis en exploitant les propriétés du noyau décrites plus haut, on peut également définir un opérateur-intégrale pour l'approximation de la dérivée du champ $f$ :

$$
\left\langle\nabla f_{i}\right\rangle=\int_{\Omega} f(x) \nabla_{x} W\left(x_{i}-x, h\right) d x
$$

qui ne fait intervenir que le gradient du noyau utilisé. Sous forme discrète, on a donc les deux formes suivantes, pour l'approximation d'un champ et de son gradient en méthode particulaire, à l'ordre 2 en espace dans le cas idéal d'une répartition cartésienne des particules :

$\left\langle f_{i}\right\rangle=\sum_{j} f\left(x_{j}\right) W\left(x_{i}-x_{j}, h\right) V_{j}$ et $\left\langle\nabla f_{i}\right\rangle=\sum_{j} f\left(x_{j}\right) \nabla_{x} W\left(x_{i}-x_{j}, h\right) V_{j}$

où $V_{j}$ représente le volume de la $j^{\text {ìme }}$ particule.

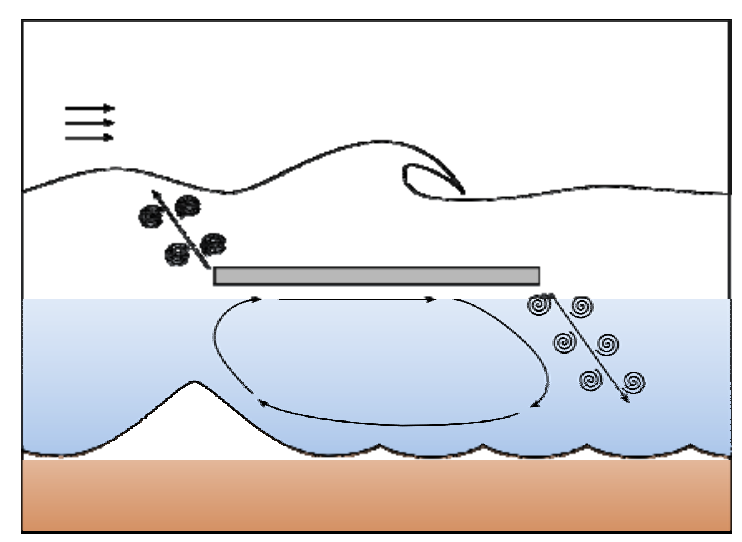

Figure 1. Représentation d'un écoulement oscillant autour d'une plaque immergée.

L'observation montre une grande diversité de phénomènes simultanés : échappements tourbillonnaires (BOULIER, 1996), déferlement, génération d'harmoniques

(BROSSARD \& CHAGDALI, 2001) et transport sédimentaire. 


\subsection{Application aux équations de la mécanique des fluides}

Les opérateurs précédents sont appliqués aux équations d'Euler pour un fluide incompressible en description lagrangienne :

$$
\left\{\begin{array}{l}
\frac{D x}{D t}=u \\
\frac{D u}{D t}=-\frac{\nabla P}{\rho}+g \\
\nabla \cdot u=0
\end{array}\right.
$$

Où $u$ représente la vitesse d'une particule fluide de position $x, P$ la pression, $\rho$ la masse volumique et $g$ l'accélération de la pesanteur. La première équation représente le déplacement lagrangien des particules, la seconde, qui correspond à l'équation de conservation de la quantité de mouvement, décrit l'évolution de la vitesse de la particule soumise à un champ de pression et à l'action de la gravité. La dernière équation traduit la contrainte d'incompressibilité du fluide. L'approche la plus courante en méthode SPH consiste à résoudre les équations du fluide, considéré faiblement compressible $($ Mach $<10)$, et de clore le système en utilisant une loi d'état pour la pression (voir équations (8)). A cet effet, les paramètres de la loi d'état sont définis de la manière suivante: $\rho_{0}$ est la masse volumique du fluide au repos, dans le cas de l'eau $\rho_{0}=1000 \mathrm{~kg} / \mathrm{m}^{3}$; la vitesse artificielle du son $c_{0}$ est définie à 10 fois la vitesse du son maximale attendue dans le fluide, le plus souvent choisie à $u_{\max }=2 \sqrt{g H}$ où $H$ représente la hauteur de la masse d'eau dans le cas présenté ci-après. Ainsi, l'équivalence (9) assure des variations de masse volumique au cours des simulations inférieures à $1 \%$.

$$
\left\{\begin{array}{l}
\frac{D x}{D t}=u \\
\frac{D u}{D t}=-\frac{\nabla P}{\rho}+g \\
\frac{D \rho}{D t}=-\rho \nabla \cdot u \\
P=\frac{\rho_{0} c_{0}^{2}}{7}\left(\left(\frac{\rho}{\rho_{0}}\right)^{7}-1\right) \\
\frac{\delta \rho \mid}{\rho} \approx \frac{u_{\max }^{2}}{c_{0}^{2}}
\end{array}\right.
$$

En appliquant les opérateurs (6) au système (8), et après quelques manipulations permettant d'assurer la conservation et la consistance du schéma, on obtient le schéma discret à intégrer en temps (par un schéma de Runge-Kutta d'ordre 2 ou supérieur) : 


$$
\left\{\begin{array}{l}
\frac{D x_{i}}{D t}=u_{i} \\
\frac{D u_{i}}{D t}=-\frac{1}{\rho_{i}} \sum_{j}\left(P_{j}+P_{i}\right) \nabla_{x_{i}} W\left(x_{i}-x_{j}, h\right) V_{j}+g \\
\frac{D \rho_{i}}{D t}=-\rho_{i} \sum_{j}\left(u_{j}-u_{i}\right) \nabla_{x_{i}} W\left(x_{i}-x_{j}, h\right) V_{j} \\
P_{i}=\frac{\rho_{0} c_{0}^{2}}{7}\left(\left(\frac{\rho_{i}}{\rho_{0}}\right)^{7}-1\right)
\end{array}\right.
$$

2.3 Exemple de validation : effondrement d'une colonne d'eau

Ce cas, couramment appelé "rupture de barrage" décrit l'évolution d'une masse fluide, de dimensions $H \times L$ initialement au repos qui, sous l'effet de la gravité, s'écroule et se propage vers un mur vertical situé à une distance $l$ à l'extrémité opposée du domaine de calcul (voir figure 2).

Les conditions aux limites (glissement) sont imposées à l'aide de "particules fantômes" crées à chaque itération, par symétrie des particules de fluide par rapport aux parois. Leurs propriétés physiques (vitesse, pression) sont déduites directement de la particule d'origine et ces particules interviennent dans les équations précédentes. La surface libre ne nécessite aucun traitement spécifique, en raison de la représentation lagrangienne de l'écoulement. Ceci est un atout de la méthode SPH par rapport à d'autres méthodes numériques telles que la méthode VOF (Volume Of Fluid) qui nécessitent différents algorithmes pour le suivi et le traitement des reconnections de surface libre. Les résultats obtenus ici concordent avec ceux de (COLAGROSSI \& LANDRINI, 2003).

\subsection{Optimisation et parallélisation}

Le code de calcul développé ici pour les applications génie côtier a fait l'objet de plusieurs optimisations. La première concerne la partie la plus gourmande en temps de calcul de l'algorithme (environ 60\%) : la détection des particules situées à une distance inférieure de $3 h$ de la particule d'intérêt, qui seront, en raison de la compacité du noyau, les seules à intervenir dans les équations (10). Cette recherche de particules proches est réalisée en superposant une grille cartésienne dont chaque maille, de taille $3 h$, contient une liste des particules présentent dans cette maille. Ainsi, pour une particule donnée, il n'est pas nécessaire de calculer les distances la séparant de toutes les autres particules, mais uniquement celles contenues dans les 8 cases adjacentes et celle contenant la particule.

D'autre part, la propriété de compacité du noyau a également été exploitée pour la parallélisation du code de calcul, en autorisant un découpage en sous-domaines indépendants. Les seules communications nécessaires entre processeurs concernent les 
particules situées à une distance inférieure à $3 h$ d'une frontière de sous-domaine (figure 3). Un algorithme de répartition dynamique de charge a également été mis en place afin d'assurer l'équilibre en temps de calcul des processeurs. Les sous-domaines varient ainsi en taille pour conserver un nombre équivalent de particules (figure 4).

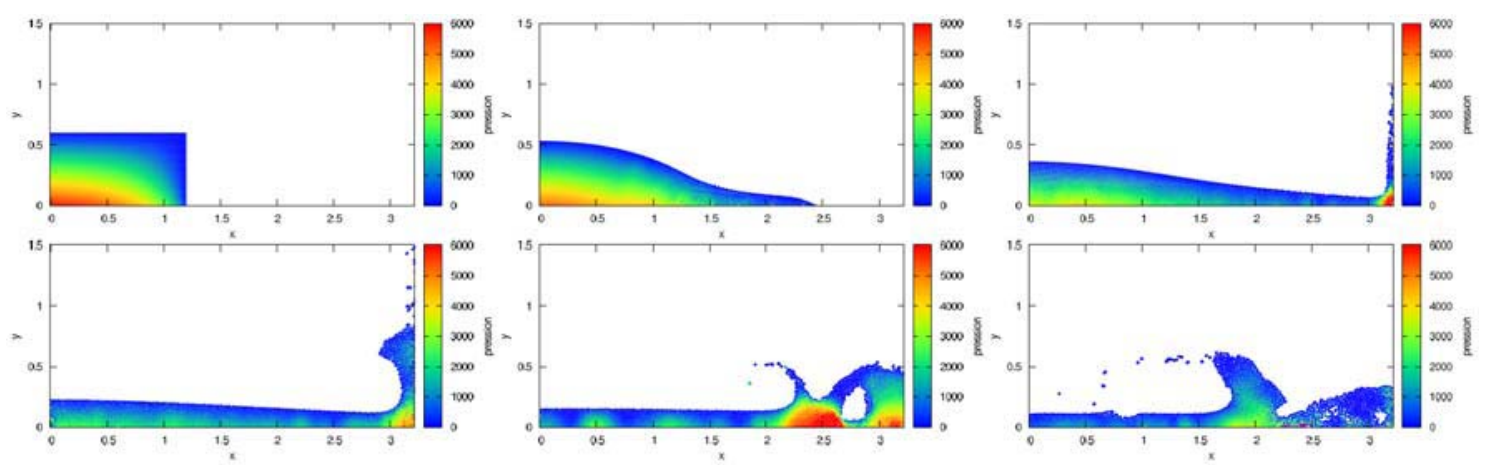

Figure 2. Simulation d'une rupture de barrage avec SPH, avec $H=0,6 \mathrm{~m}$ et $L=1,2 \mathrm{~m}, l=3,2196 \mathrm{~m}:$ de gauche à droite et de haut en bas, solution initiale $\grave{a} t=0^{+} s$, écoulement du fluide sous l'action de la gravité à $t=0,425 \mathrm{~s}$, impact à $t=0,85 \mathrm{~s}, t=1,275 \mathrm{~s}, t=1,7 \mathrm{~s}$ et $t=2,125 \mathrm{~s}$

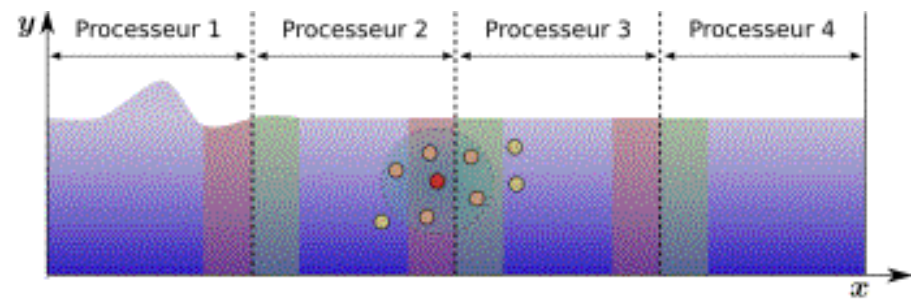

Figure 3. Principe de la répartition en sous-domaines, seules les zones vertes et rouges nécessitent l'envoi d'informations aux processeurs voisins.
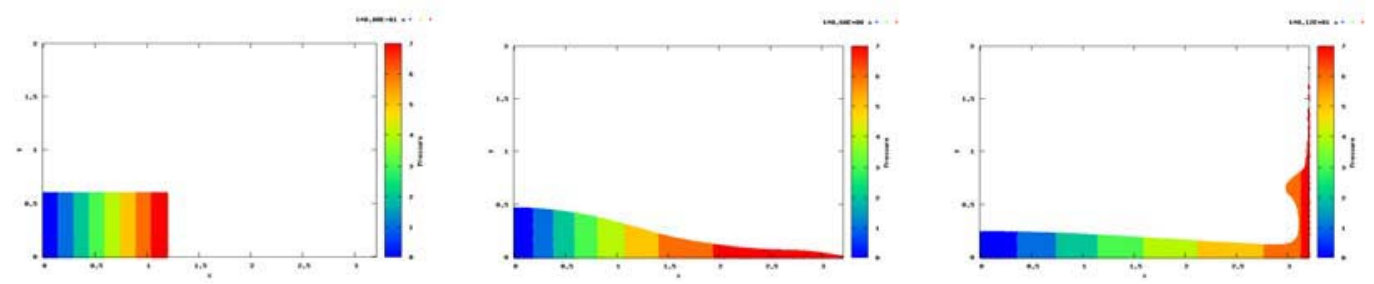

Figure 4. Evolution des sous-domaines dans le cas de la rupture de barrage.

La figure 5 montre d'une part une comparaison de nos résultats avec des données expérimentales pour la pression mesurée à l'impact du front d'eau sur le mur vertical. On observe un bon accord. D'autre part, la figure 5 montre également l'accélération du code parallèle par rapport au code séquentiel. Pour un grand nombre de particules, cet algorithme de parallélisation est efficace quelque soit le nombre de processeurs utilisés. Sur cet exemple de la rupture de barrage, la simulation de 2s de temps réel avec 4900 
particules sur 4 processeurs (Intel Xeon Quad 1.6 GHz) requiert environ 10 minutes de calcul. La complexité algorithmique du modèle implique que les temps de calcul augmentent exponentiellement avec le nombre de particules. Ainsi, pour une simulation de canal à houle contenant 50000 particules sur huit processeurs, 24 heures de calcul sont nécessaires pour atteindre 2 minutes de temps réel.

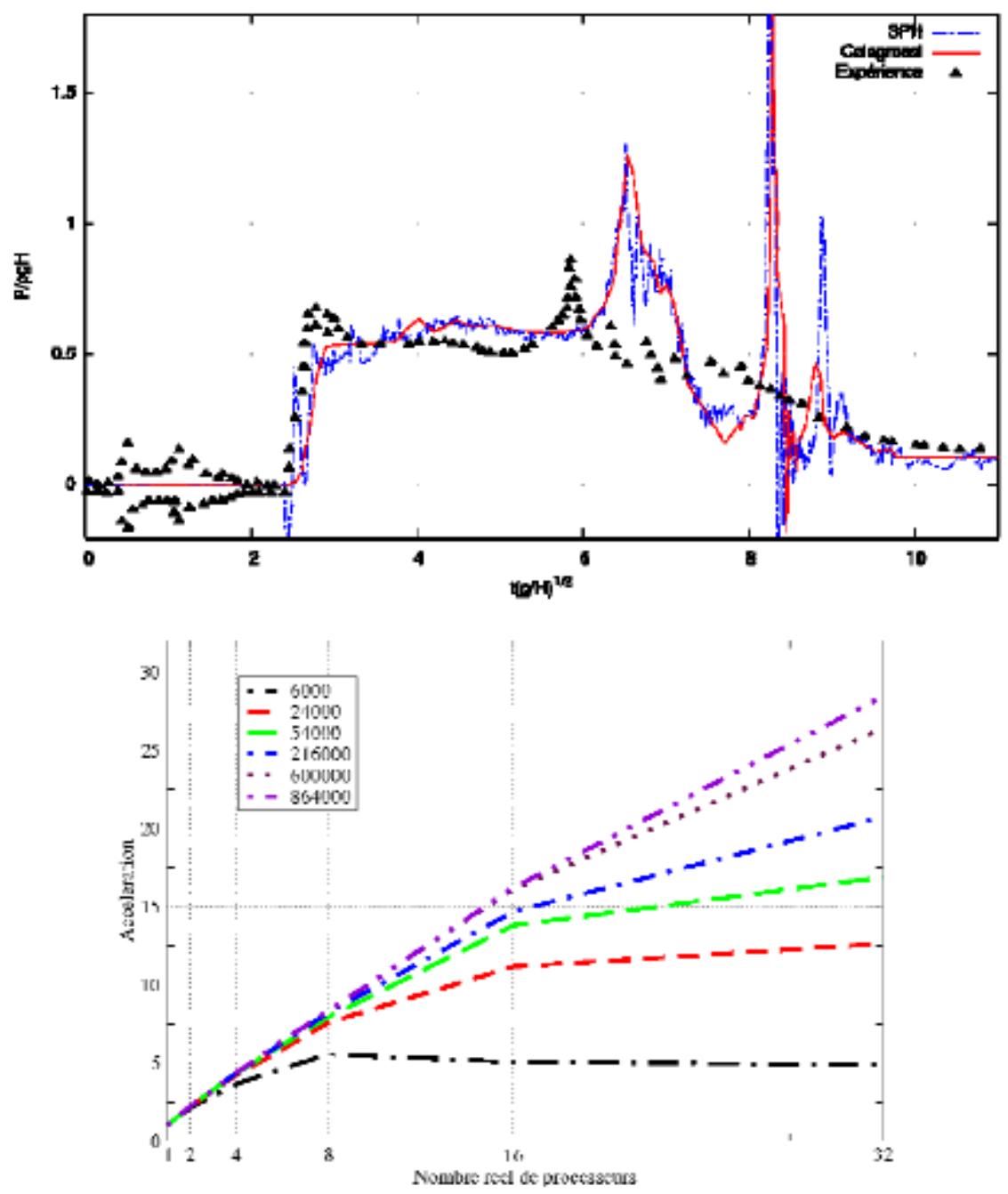

Figure 5. Pression à l'impact sur le mur vertical (haut). Accélération du code de calcul permise par cette parallélisation (bas).

\section{Amortissement d'une houle par une plaque immergée en canal}

Pour la protection des côtes, une plaque immergée est une solution économique et discrète. La houle incidente est partiellement réfléchie, générant une houle plus complexe en amont et en aval de la plaque (BROSSARD \& CHAGDALI, 2001). Le cisaillement ainsi généré aux extrémités est à l'origine d'échappements tourbillonnaires qui peuvent interagir avec le lit du canal et la surface libre selon la position de la plaque. 
Le sujet a déjà été étudié en considérant l'écoulement irrotationnel (PATARAPANICH \& CHEONGH, 1989).

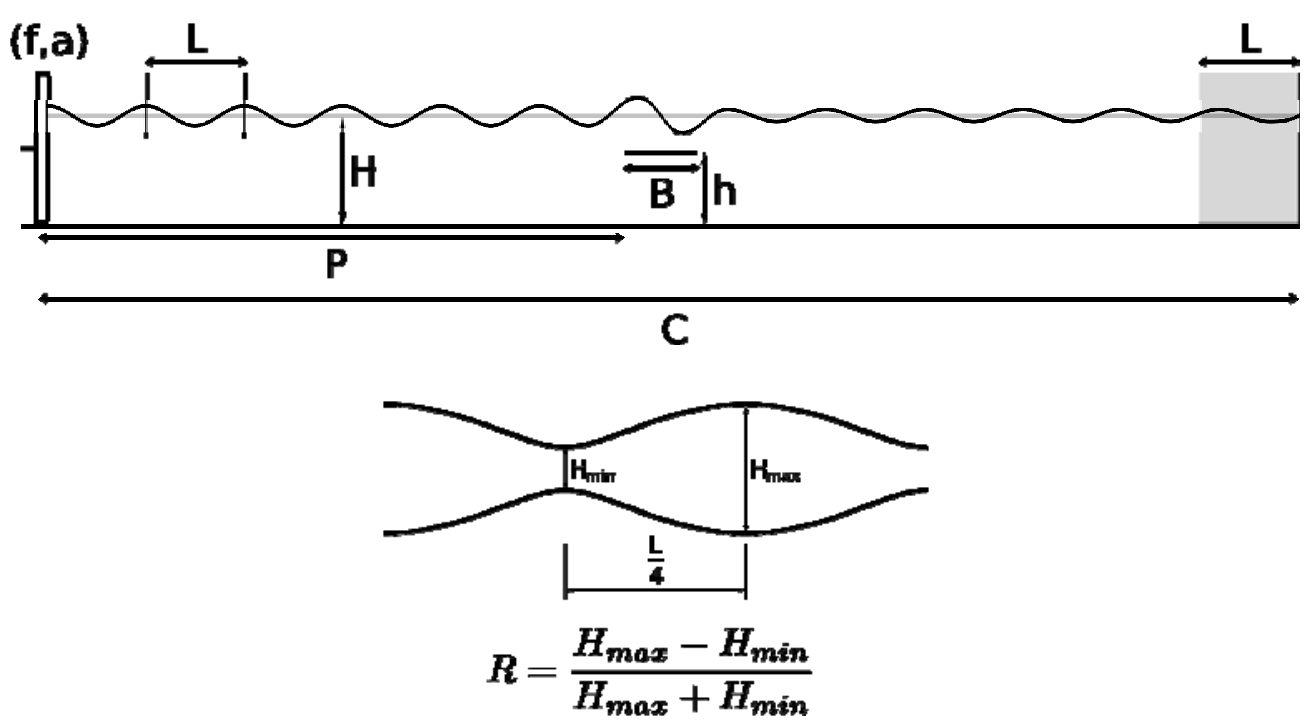

Figure 6. Paramètres du problème: $H=0,2 m, C=5 m, P=2 m, B=0,25 m$ et $h=0,15 m$. Le batteur piston (à gauche) génère une houle sinusoïdale de fréquence f amortie sur la dernière longueur d'onde du canal. L'amplitude du batteur est a=0,005m. Enveloppe de surface libre calculée dans le cas $f=1,224 \mathrm{~Hz}$.

Par des approches à la fois expérimentale et numérique (en méthode Vortex), (BOULIER \& BELORGEY, 1994 ; BOULIER, 1996 ; POUPARDIN et al., 2010) se sont intéressés à la dynamique de tourbillons générés aux extrémités de la plaque. Il existe ainsi plusieurs façons de prédire l'amortissement de la houle par un corps immergé mais aucune approximation linéraire de ce problème ne permet la prise en compte de l'interaction de l'obstacle avec le fond ou la surface libre. La simulation de cet écoulement complexe nécessite une modélisation robuste des conditions limites.

La figure 6 expose les paramètres géométriques choisis, en négligeant dans un premier temps les effets visqueux. L'élévation de la surface libre a été enregistrée en amont de la plaque par plusieurs sondes numériques équidistantes. L'enveloppe de surface ainsi déterminée permet, par la méthode des ventres et des nœuds, de déterminer le taux de réflexion de la plaque (figure 7).

Les résultats obtenus $(\mathrm{SPH})$ sont représentés par la figure 7, montrant un bon accord avec les données expérimentals (EXP) (PERRET \& BROSSARD, 2009) . Un maximum est obtenu quand la longueur d'onde de houle estimée au dessus de la plaque correspond à deux fois la longueur de celle-ci. La solution analytique (ANA) présentée ici est basée sur un modèle potentiel de l'écoulement. Elle a tendance à surestimer le taux de réflexion pour les faibles longueurs d'ondes de houle incidente et à le sous-estimer dans le cas des plus grandes longueurs d'ondes. 


\section{XI $I^{\text {èes }}$ Journées Nationales Génie Côtier - Génie Civil}

Les Sables d'Olonne, 22-25 juin 2010

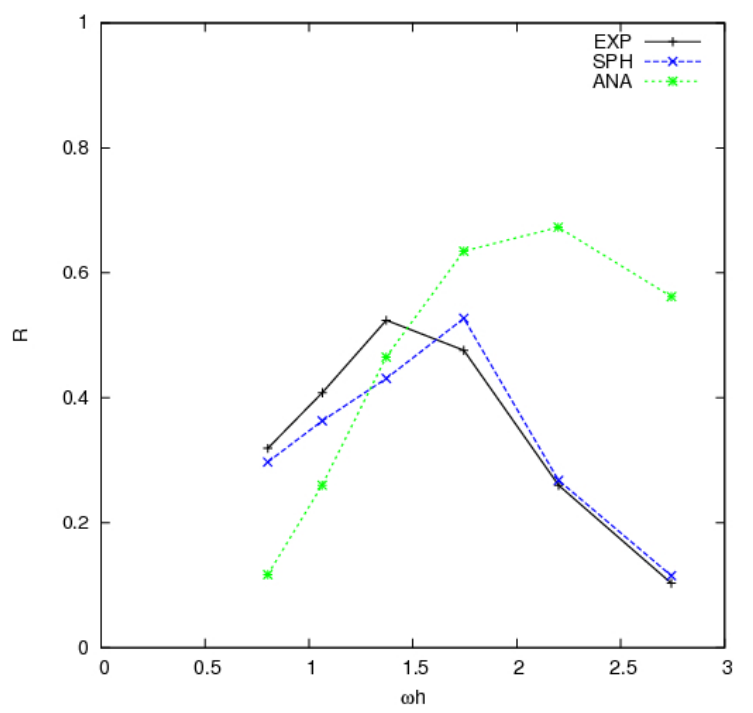

Figure 7. Taux de réflexion de la houle $R$ en fonction du rapport $\omega / \mathrm{h}$ avec $\omega$ la pulsation du batteur. Les valeurs correspondantes en fréquence sont: $f=0,814 \mathrm{~Hz}$, $1,02 \mathrm{~Hz}, 1,224 \mathrm{~Hz}, 1,428 \mathrm{~Hz}, 1,633 \mathrm{~Hz}$ et 1,839 $\mathrm{Hz}$.

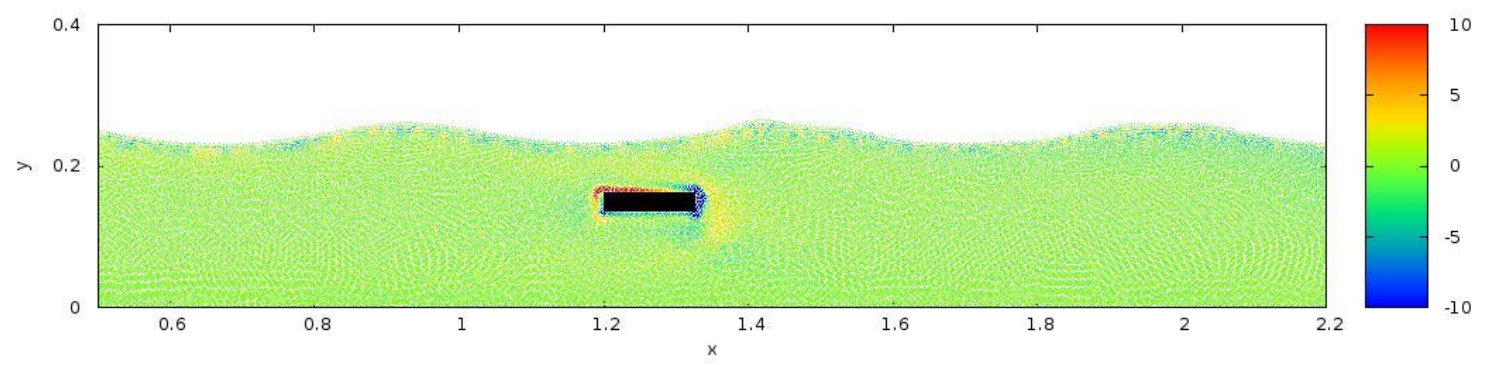

Figure 8. Champ de vorticité autour d'une plaque immergée (voir figure 5) avec $B=0,125 \mathrm{~m}$ et d'épaisseur 0,025 m.

Dans un second temps, la viscosité du fluide a été prise en compte. La condition à imposer sur le contour de la plaque devient alors une condition d'adhérence. Celle-ci a été modélisée par la technique de frontière immergée, étendue à la méthode SPH. Le rotationnel de vitesse généré par la présence de la plaque est représenté sur la figure 8 .

\section{Conclusion}

Un modèle lagrangien basé sur la méthode SPH a été présenté pour simuler les écoulements de fluides visqueux à surface libre. La durée de ces simulations a été réduite par l'optimisation et la parallélisation du code de calcul. Enfin, la prise en compte de géométries complexes pour la simulation d'interaction houle-corps immergés est également possible, par la méthode de frontière immergée. 
Cet outil devrait ainsi permettre d'observer et de quantifier les efforts auxquels sont soumis de telles structures marines. Il s'agit dans un premier temps d'achever la validation de l'ensemble du modèle sur un problème d'interaction de la houle avec une structure géométriquement simple. Puis l'étude d'écoulements réels, autour d'ouvrages marins aux formes complexes pourra être envisagée.

\section{Références bibliographiques}

BOULIER B., BELORGEY M. (1994). Ecoulement tourbillonnaire et zone d'affouillement générés par la houle en présence d'une plaque immergée. IIIèmes Journées Génie Côtier - Génie Civil, Sète, pp 39-45. doi:10.5150/jngcgc.1994.005-B BOULIER B. (1996). Evolution spatio-temporelle de structures tourbillonnaires dans la houle. Thèse, Université du Havre.

BROSSARD J., CHAGDALI M. (2001). Experimental investigation of the harmonic generation by waves over a submerged plate. Coastal Engineering, $\mathrm{n}^{\circ} 42$, pp 277-290. doi:10.1016/S0378-3839(00)00064-8

COLAGROSSI A., LANDRINI M. (2003). Numerical simulation of interfacial flows by smoothed particle hydrodynamics. Journal of Computational Physics, $\mathrm{N}^{\circ}$ 191, pp 448-475. doi:10.1016/S0021-9991(03)00324-3

GINGOLD R., MONAGHAN J.J. (1977). Smoothed particle hydrodynamics: Theory and application to non-spherical stars. Monthly Notices Royal Astronomy Society, $\mathrm{n}^{\circ}$ 181, pp 375-389.

LUCY L. (1977). A numerical approach to the testing of the fission hypothesis. Astronomical Journal, n 82, pp 1013-1024. doi:10.1086/112164

MONAGHAN J. (1994). Simulating free surface flows with SPH. Journal of Computational Physics, $\mathrm{n}^{\circ}$ 110, pp 399-406. doi:10.1006/jph. 1994.1034

PATARAPANICH M., CHEONG H. (1989). Reflection and transmission characteristics of regular and random waves from a submerged horizontal plate. Coastal Engineering, $\mathrm{n}^{\circ} 13$, pp 161-182. doi:10.1016/0378-3839(89)90022-7

PERRET G., BROSSARD J. (2009). Mesures expérimentales du coefficient de réflexion d'une plaque immergée dans la houle. Rapport interne du Laboratoire Ondes \& Milieux Complexes.

POUPARDIN A., BOURNETON N., PERRET G., PINON G., RIVOALEN E., BROSSARD J. (2010). Sillages tourbillonnaires des structures immergées. XIèmes Journées Nationales Génie Côtier - Génie Civil, Les Sables d'Olonne, pp 803-812. doi:10.5150/jngcgc.2010.089-P 